\title{
Advanced Multifunctional MMOD Shield: Radiation Shielding Assessment
}

\author{
Kristina Rojdev and Eric Christiansen \\ NASA-Johnson Space Center \\ 2101 NASA Parkway, Houston, TX 77058
}

\begin{abstract}
As NASA is looking to explore further into deep space, multifunctional materials are a necessity for decreasing complexity and mass. One area where multifunctional materials could be extremely beneficial is in the micrometeoroid orbital debris (MMOD) shield. A typical MMOD shield on the International Space Station (ISS) is a stuffed whipple shield consisting of multiple layers. One of those layers is the thermal blanket, or multi-layer insulation (MLI). By increasing the MMOD effectiveness of MLI blankets, while still preserving their thermal capabilities, could allow for a less massive MMOD shield. Thus, a study was conducted to evaluate concept MLI blankets for MMOD shields.
\end{abstract}

In conjunction, these MLI blankets and the subsequent MMOD shields were also evaluated for their radiation shielding effectiveness towards protecting crew. These concepts were evaluated against the ISS MLI blankets and the ISS MMOD shield, which acted as the baseline. These radiation shielding assessments were performed using the high charge and energy transport software (HZETRN). This software is based on a one-dimensional formula of the Boltzmann transport equation with a straight-ahead approximation. Each configuration was evaluated against the following environments to provide a diverse view of radiation shielding effectiveness in most space environments within the heliosphere: August 1972 solar particle event, October 1989 solar particle event, 1982 galactic cosmic ray environment (during solar maximum), 1987 galactic cosmic ray environment (during solar minimum), and a low earth orbit environment in 1970 that corresponded to an altitude of $400 \mathrm{~km}$ and inclination of $51.6^{\circ}$. Both the absorbed dose and the dose equivalent were analyzed, but the focus of the discussion was on the dose equivalent since the data is most concerned with radiation shielding of the crew.

The following paper outlines the evaluations performed and discusses the results and conclusions of this evaluation for radiation shielding effectiveness. Future work will focus on optimizing the radiation shielding properties for the MMOD shield.

Submission for the $42^{\text {nd }}$ International Conference on Environmental Systems (ICES)

July 15-19, 2012

San Diego, CA 